\title{
CHARACTERISTICS OF UROLITHIASIS IN THE DOG POPULATION OF HUNGARY FROM 2001 TO 2012
}

\author{
Balázs BENDE $^{1 *}$, Krisztina Borbála KovÁCs ${ }^{2}$, Norbert SOLYMOSI ${ }^{3}$ and Tibor NÉMETH ${ }^{2}$ \\ ${ }^{1}$ Animal Hospital of Budapest, Budapest Urolith Centre, Lehel u. 43-47, H-1135 Budapest, \\ Hungary; ${ }^{2}$ Department and Clinic of Surgery and Ophthalmology and ${ }^{3}$ Department of \\ Animal Hygiene, Herd-health and Veterinary Ethology, Faculty of Veterinary Science, \\ Szent István University, Budapest, Hungary
}

(Received 1 December 2014; accepted 22 June 2015)

The objective of this study was to describe the epidemiology of canine urolithiasis in Hungary in order to determine the annual incidence of urolithiasis and to identify breeds at risk for different types of urolithiasis. Data of a total of 2,543 canine uroliths analysed in the laboratory of the Budapest Urolith Centre were evaluated retrospectively from 2001 to 2012. Logistic regression was used to assess odds ratios for the proportion of each affected breed compared to those of crossbreeds. The annual incidence of urolithiasis was evaluated by the number of submissions compared to the estimated number of dogs in the population from which the samples originated. Epidemiologic data revealed a relatively high and increasing proportion of struvite urolithiasis. Statistical analysis of breed predispositions resulted in the detection of breeds not having been reported at risk (e.g. Bernese Mountain dog - struvite, Bichon Frise, Bolognese, Tibetan Terrier purine, French Bulldog - cystine). Conflicting results were revealed for some other breeds previously described as being affected by certain types of urolithiasis (Chihuahua, Pekingese, Shih Tzu, English Cocker Spaniel). Regardless of the type of urolithiasis, its average cumulative incidence in the dog population of Hungary was found to be $1.76 / 10,000 /$ year.

Key words: Urolithiasis, epidemiology, incidence, dog, breed predisposition

Several different studies have been published on the epidemiology of canine urolithiasis over the past decades (Table 3). Differences in breed predispositions in different geographic regions and changes over time in the proportion of different types of uroliths have also been identified by others (Ling et al., 2003; Lulich et al., 2013). Breeders' activity or owners' breed preferences also have an influence on the epidemiology of urolithiasis. Generational changes in the dog population may cause shifts in breed proportions and have a further impact on the epidemiological data. New or periodically repeated analyses can estimate the

*Corresponding author; E-mail: bende@buc.hu; Phone and Fax: 0036 (1) 350-0361 
effect of this change and new considerations may alter the focus of investigations or interventions (dietary strategy, clinical screening or change in breeding policy) to be done. The aim of this study was to describe the epidemiology of canine urolithiasis in Hungary, where the dog population had not been evaluated in this regard before. Based upon the epidemiological data the authors intended to determine the incidence of urolithiasis and to identify breeds at risk for different types of urolithiasis within this population.

\section{Materials and methods}

An analysis of a total of 2,543 canine uroliths sent to the laboratory of the Budapest Urolith Centre (BUC) was conducted in Hungary with a standard data questionnaire containing breed, age, gender and clinical data from 2001 to 2012. After macro- and microscopic evaluation of the samples a quantitative or semiquantitative evaluation of the chemical composition of each different layer of uroliths was performed by the ultramicrochemical (UMC) method (Reanal, Hungary; Berényi and Frang, 1989; Bruckner et al., 1989) and in part by infrared spectroscopy (Perkin Elmer 1600 FTIR). Uroliths were classified on the basis of the predominant mineral component that exceeded $70 \%$ of the stone mass. The proportion of affected breeds for each urolith type was compared to the proportion of crossbreed dogs. Odds ratio (OR) and significance level were calculated by logistic regression using the $\mathrm{R}$ statistical software (R Core Team, 2013). A unique model was fitted for each urolith type. The breed was used as independent variable in the models. The level of statistical significance was set at $5 \%(\mathrm{P}<$ $0.05)$. Population data were gathered from a previous report on the dog population of Budapest ( $\mathrm{n}=124,459$; Bende et al., 2003) and from the Hungarian Microchip Register $(\mathrm{n}=543,929)$. These two datasets were taken into account to calculate the change of breed composition of the dog population between the start and the end of the study period. The number of dogs living in the countryside was estimated from data of the registered numbers of immunisation against rabies provided by the Animal Welfare Directorate of the National Food Chain Safety Office (NFCSO) in the years 2010, 2011 and 2012, respectively.

The cumulative incidence of canine urolithiasis was estimated from the numbers of canine sample submissions to the BUC and the data given by the NFCSO yearly.

\section{Results}

Distribution of uroliths by type, gender and breed (Tables 1 and 2)

Cumulative incidence of urolithiasis in the population. According to data on the registered rabies vaccinations in Hungary, the number of such vaccina- 
tions was 1,415,699, 1,369,000 and 1,387,600 in 2010, 2011 and 2012, respectively. The laboratory received 240, 236 and 260 samples, respectively, from dogs from the entire country in the same period. The calculated incidence of urolithiasis was $1.69,1.72$ and $1.87 / 10,000 /$ year, respectively. The average of the cumulative incidence for the three-year period was 1.76/10,000.

Change of the distribution of urolith types between 2001 and 2012. The proportion of struvite urolithiasis increased from $54.4 \%$ to $68.8 \%$, while that of purine uroliths decreased from $22.0 \%$ to $8.1 \%$. During the study period there was no observable tendency in the change of proportion of oxalate and cystine stones (Table 4). The ratio of struvite to oxalate uroliths increased (Fig. 1).

Breed prevalence in the population, with special regard to the breeds most affected by urolithiasis. In the 12 years evaluated in this study the composition of the dog population inevitably changed significantly. These changes influenced the prevalence of different types of urolithiasis. In order to evaluate changes in the population, two databases of the dog population (the dog population of $\mathrm{Bu}-$ dapest and the Hungarian Microchip Register) were compared with special emphasis on the change of proportions of the most affected breeds. Although a total of 99 breeds were involved in the study, $80 \%$ of the samples came from only 23 breeds. Sixteen of them were characterised as breeds being at an increased risk for at least one type of urolithiasis (Table 2). Changes in the average annual proportions of the 23 most affected breeds between the first and the last year of the study were compared to changes in the proportions of the same breeds in the population (Fig. 2). The proportion of dogs affected by urolithiasis increased remarkably in Pug (from 0.3 to 4), Bolognese (0.3 to 2), Bichon Frise (0.3 to 1), English Bulldog (0.9 to 2.4) and Yorkshire Terrier (3.8 to 11.1), while the representation of other breeds such as the Dalmatian (from 15.6 to 1), Black Russian Terrier (2.1 to 0.4), Poodle (1.8 to 0.4) and Basset Hound (1.2 to 0.4) decreased prominently. The direction of change in the dog population affected by urolithiasis was mostly the same as in the general dog population, with some exceptions: there was an opposite change concerning the Basset Hound, Chihuahua, Miniature Schnauzer, Pekingese and Puli.

\section{Discussion}

\section{Prevalence of the different types of urolithiasis}

Although several studies have already been conducted to identify the patterns of the urolithiasis in the USA, Canada and Europe, this is the first retrospective epidemiological evaluation of canine urolithiasis in Hungary. The distributions of different urolith types found in other studies and our results are summarised in Table 3. 
BENDE et al.

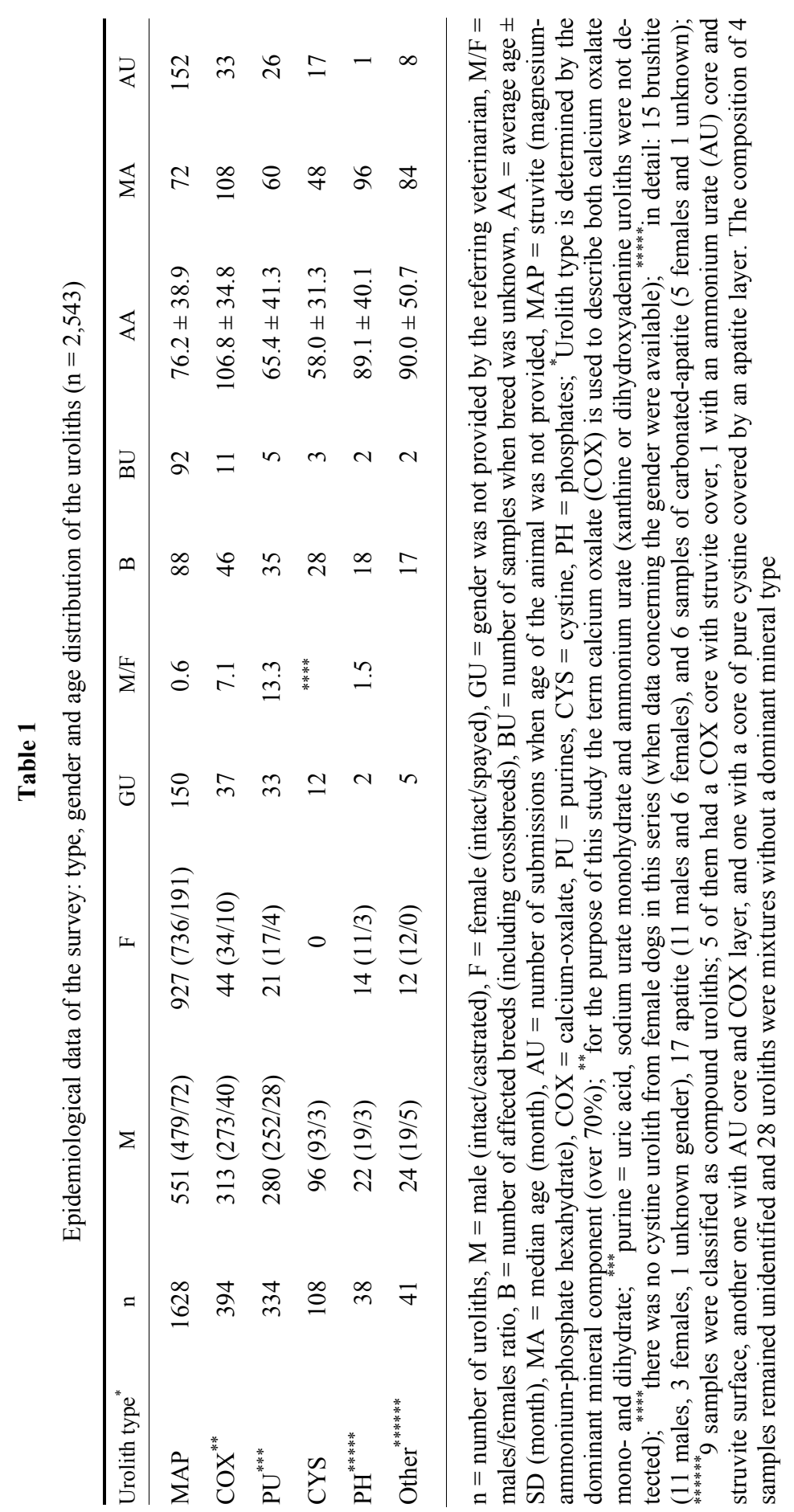


Table 2

Breeds affected by different kinds of urolithiasis in the population

\begin{tabular}{|c|c|c|c|c|c|}
\hline & Breed at risk & $\mathrm{n} / \mathrm{N}$ & OR & $95 \% \mathrm{CI}$ & $\mathrm{P}$ \\
\hline \multirow[t]{5}{*}{ Struvite } & Bernese Mountain Dog & $31 / 32$ & 11.1 & $1.5-82.7$ & 0.018 \\
\hline & German Shepherd Dog & $49 / 51$ & 8.8 & $2.1-36.9$ & 0.002 \\
\hline & Shih Tzu & $77 / 88$ & 2.5 & $1.2-4.9$ & 0.007 \\
\hline & English Cocker Spaniel & $89 / 105$ & 2.0 & $1.1-3.5$ & 0.019 \\
\hline & Crossbreed & $259 / 352$ & ref. group & ref. group & ref. group \\
\hline \multirow[t]{5}{*}{$\operatorname{COX}$} & Miniature Pinscher & $10 / 20$ & 5.4 & $2.1-13.5$ & $<0.001$ \\
\hline & Yorkshire terrier & $84 / 212$ & 3.5 & $2.3-5.2$ & $<0.001$ \\
\hline & Miniature Poodle & $9 / 24$ & 3.2 & $1.3-7.7$ & 0.008 \\
\hline & Miniature Schnauzer & $26 / 89$ & 2.2 & $1.3-3.8$ & 0.003 \\
\hline & Crossbreed & $55 / 352$ & ref. group & ref. group & ref. group \\
\hline \multirow[t]{9}{*}{ Purine } & Dalmatian & $151 / 160$ & 352.3 & $152.3-815.3$ & $<0.001$ \\
\hline & Black Russian Terrier & $27 / 29$ & 283.5 & $61.9-1298.0$ & $<0.001$ \\
\hline & Bulldog & $26 / 58$ & 17.1 & $8.3-35.1$ & $<0.001$ \\
\hline & Tibetan Spaniel & $3 / 8$ & 12.6 & $2.8-57.4$ & 0.001 \\
\hline & Giant Schnauzer & $2 / 8$ & 7.0 & $1.3-37.4$ & 0.022 \\
\hline & Bolognese & $6 / 25$ & 6.6 & $2.3-18.9$ & $<0.001$ \\
\hline & American Staffordshire Terrier & $4 / 29$ & 3.4 & $1.0-10.8$ & 0.042 \\
\hline & Yorkshire terrier & $27 / 212$ & 3.1 & $1.6-5.8$ & $<0.001$ \\
\hline & Crossbreed & $16 / 352$ & ref. group & ref. group & ref. group \\
\hline \multirow[t]{9}{*}{ Cystine } & Basset Hound & $19 / 37$ & 40.2 & $16.0-101.3$ & $<0.001$ \\
\hline & Bulldog & $19 / 58$ & 18.6 & $7.9-43.9$ & $<0.001$ \\
\hline & Rottweiler & $8 / 30$ & 13.9 & $4.9-39.4$ & $<0.001$ \\
\hline & Miniature Pinscher & $5 / 20$ & 12.7 & $3.8-42.6$ & $<0.001$ \\
\hline & French Bulldog & $3 / 14$ & 10.4 & $2.5-43.8$ & 0.001 \\
\hline & Wirehaired Dachshund & $2 / 12$ & 7.6 & $1.5-39.9$ & 0.016 \\
\hline & Dachshund & $13 / 89$ & 6.5 & $2.7-15.8$ & $<0.001$ \\
\hline & Chihuahua & $3 / 27$ & 4.8 & $1.2-18.8$ & 0.025 \\
\hline & Crossbreed & $9 / 352$ & ref. group & ref. group & ref. group \\
\hline
\end{tabular}

$\mathrm{n}=$ number of dogs affected by the given type of urolithiasis; $\mathrm{N}=$ number of all individuals in the same breed affected by any kind of stone disease in the series, OR $=$ Odds ratio, $95 \% \mathrm{CI}=$ the lower and upper values of the $95 \%$ confidence interval given by the statistical analysis by the means of logistic regression, $\mathrm{P}=\mathrm{P}$ values. Breeds affected by more than one type of urolithiasis are indicated in italics

Breed predisposition. The over- (or under-) representation of a breed among dogs affected by a certain type of urolithiasis is influenced by either predisposing or protecting factors (thought to be genetically determined alterations) and, furthermore, by either the relatively high or low number of individuals of that breed in the overall population. Comparison of the breed proportions to crossbreed dogs with respect to any kind of urolithiasis is a reasonable way to demonstrate the presence or absence of suspected predisposing (or protecting) 


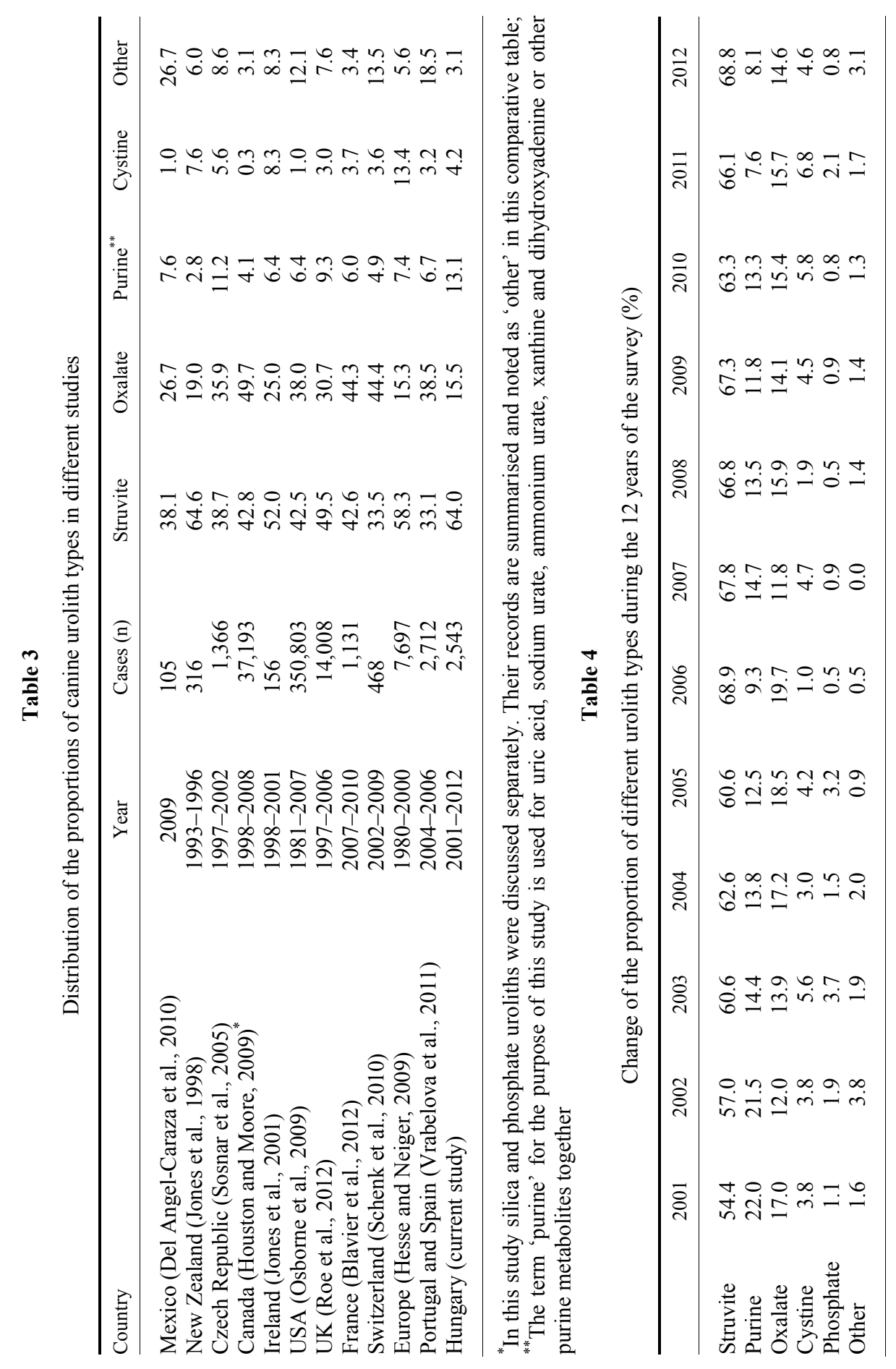




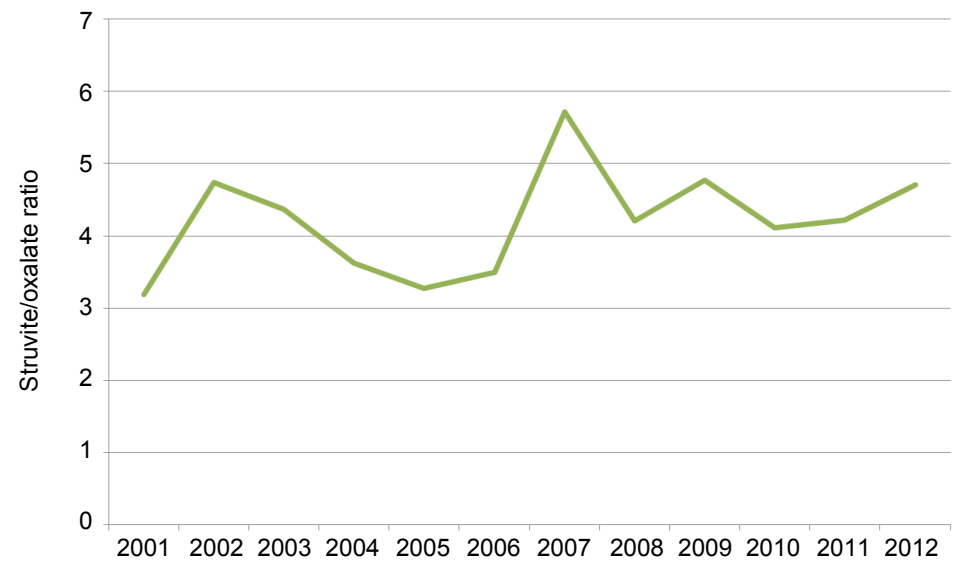

Fig. 1. Struvite/oxalate ratio

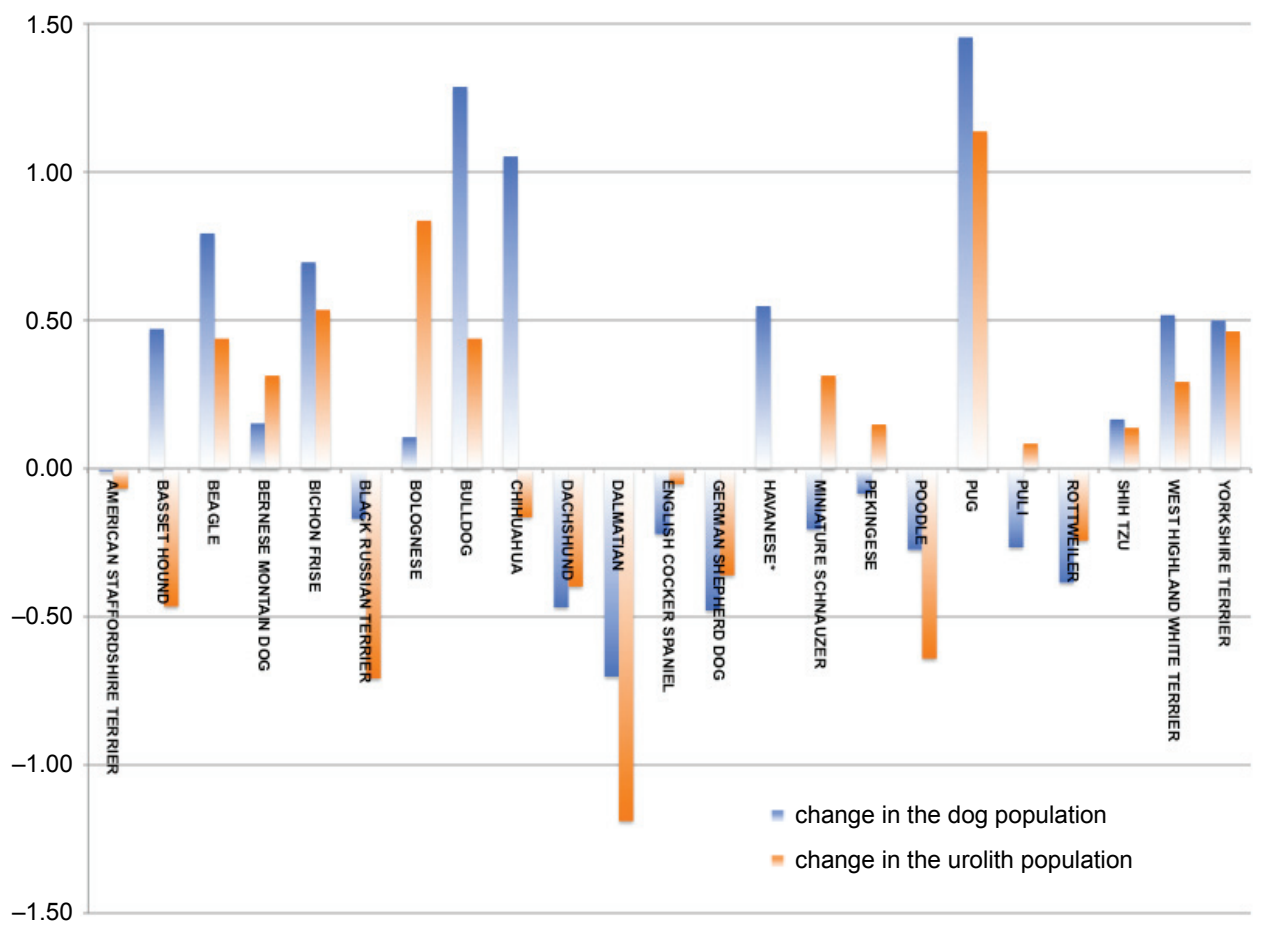

Fig. 2. Observed change of the proportion of the most affected 23 breeds in the urolith database and in the dog population; Bars represent in a logarithmic scale how many times the proportion of a breed increased or decreased from the start to the end of the study period. *Havanese dogs were not recorded in the first years of the survey but 22 were counted in the last two years. Thus, the proportion increased from 0 to $4.4 \%$, and this change cannot be depicted as a ratio 
factors. Considerations on breed predispositions are not necessarily valid for all geographic regions and periods. Conflicting data on breeds predisposed or not predisposed to a certain type of urolithiasis can be observed when one compares the results of other studies reported in the literature: for example the Border Collie was found to be at risk of urolithiasis by authors of a survey conducted in the UK (Roe et al., 2012), while the same breed was found to be characterised by a relatively low risk in the USA (Low et al., 2010). Current considerations on breed predispositions also have to be taken into account with caution; thus, they are valid only at the time and place of the study.

Struvite urolithiasis in dogs are mainly caused by bacterial infection in the urinary tract (Hesse and Neiger, 2009). If there were no other factor predisposing to struvite urolithiasis, the prevalence of breeds among the struvite-forming dogs would reflect the prevalence of the given breeds in the population. Nevertheless, some breeds affected by struvite urolithiasis show higher proportions than would be expected from its representation in the population (see below). Although increased susceptibility of the urinary tract to infection is suspected, the aetiology is still unknown. According to the results of our study, Shih Tzus and English Cocker Spaniels were found to be characterised by a high prevalence of struvite urolithiasis, similarly as it has been reported by others (Picavet et al., 2007; Rogers et al., 2011; Vrabelova et al., 2011; Blavier et al., 2012; Roe et al., 2012). However, our data showed significantly lower OR values in some other breeds regarded as breeds being at high risk for struvite urolithiasis such as the Miniature Schnauzer (Vrabelova et al., 2011; Roe et al., 2012) and the Dachshund (Sosnar et al., 2005). The prevalence of struvite urolithiasis in German Shepherds has not been reported yet; however, evaluation of the representation of this breed in our series revealed a high OR value at the highest significance level. The authors observed a completely unique appearance of struvite urolithiasis in Bernese Mountain dogs. Males were four times more affected than females and the mean age of affected individuals was 33 months (median: 12). Although the relatively high frequency of that breed among struvite-forming dogs was detected in a European study (Hesse and Neiger, 2009), the uncommon pattern of this phenomenon was not discussed. Others also reported a more frequent occurrence of struvite urolithiasis in younger dogs (Low et al., 2010; Roe et al., 2012). The reasons for these peculiar conditions remain unknown and need further investigation.

Concerning calcium oxalate urolithiasis, the mean age was the highest among stone-forming dogs as has also been observed in other studies (Lekcharoensuk et al., 2000; Wisener et al., 2010a). The fact that calcium excretion increases with age makes it a suspected predisposing factor (Lulich et al., 2013). Breeds found to be at a higher risk included, in partial agreement with the results of other studies, the Miniature Pinscher (Blavier et al., 2012), the Miniature Schanuzer (Blavier et al., 2012; Roe et al., 2012; Lulich et al., 2013), the York- 
shire Terrier (Picavet et al., 2007; Roe et al., 2012; Lulich et al., 2013) and the Miniature Poodle (Sosnar et al., 2005; Picavet et al., 2007).

The breed Shih Tzu has been found to be at risk of both calcium oxalate and struvite urolithiasis in several studies (Low et al., 2010; Vrabelova et al., 2011; Roe et al., 2012) except one (Picavet et al., 2007) in which this breed was found to be at risk of struvite rather than calcium oxalate urolithiasis. In our study, the Shih Tzu was found to be at a higher risk of struvite urolith formation. Other breeds that have already been mentioned by others as breeds at a higher risk of calcium oxalate urolithiasis, such as the Chihuahua (Roe et al., 2012), the Pekingese (Vrabelova et al., 2011) and the English Cocker Spaniel (Vrabelova et al., 2011) were not found to be predisposed and even showed expressively low OR values in the present study.

Dalmatian dogs are affected by purine stone formation due to a genetic defect affecting purine metabolism (hyperuricosuria, HUU) (Low et al., 2010; Roe et al., 2012). In the current study, beside the Dalmatian breed, Black Russian Terrier (BRT), English Bulldog, Tibetan Spaniel, Bichon Frise, Bolognese and Yorkshire Terrier were characterised by a significantly higher risk of purine urolithiasis compared to crossbreeds. Affection of the BRT by this type of urolithiasis has already been reported (Bende and Németh, 2004), and HUU has been identified as the underlying condition (Karmi et al., 2010). The predisposition of English Bulldogs and Yorkshire terriers has also been reported (Vrabelova et al., 2011; Roe et al., 2012). The Yorkshire Terrier is affected by inherited portosystemic shunts that can lead to an increased frequency of purine urolith formation (Tobias and Rohrbach, 2003). English Bulldogs are suspected to be predisposed to HUU (Bannasch and Henthorn, 2009). To our knowledge, Tibetan Spaniels and Bolognese dogs have not been observed previously as breeds at risk of purine stone formation or any other above-mentioned hereditary underlying disorder.

The high risk of cystine stone formation in Basset Hounds, Dachshunds, Miniature Pinschers and English Bulldogs has already been reported (Jones et al., 2001; Sosnar et al., 2005; Blavier et al., 2012). Irish Terriers are known to be highly predisposed to cystinuria (Brons et al., 2013). In the present study, all three samples from Irish Terriers were pure cystine stones. The OR value compared to crossbreeds was very high (over 100) without statistical significance. All the samples from Irish Terrier dogs came from intact males. Although the mean age of Irish Terriers and Basset Hounds was 66 months and 55 months, respectively, cystine urolith formation in English Bulldogs was observed in relatively young individuals: the mean age was 40 months and the youngest individual was 16 months old. French Bulldogs were previously not reported to be at risk of cystine urolithiasis. Surprisingly, the two reportedly most affected breeds, i.e. the Newfoundland dog and the Labrador Retriever (Henthorn et al., 2000; Bannasch and Henthorn, 2009), were not represented in our series except for a single sample submitted from a Labrador Retriever. Our results suggest, accord- 
ing to the recently published classification system (Brons et al., 2013), that Type III androgen-dependent cystinuria may be the most common form in our dog population as only male individuals were affected.

\section{Incidence of urolithiasis}

The incidence of urolithiasis was estimated from the ratio of the number of submitted samples and the registered number of dogs in the years from 2010 to 2012. In Hungary all dogs have to be vaccinated yearly against rabies. The execution of this vaccination has to be verified by the veterinarian with a holographic sticker inserted into the vaccination book. The number of stickers used for this purpose is recorded by the NFCSO. Since there was no other laboratory specialised in urolith analysis in Hungary, the incidence of urolithiasis could reliably be estimated from the number of submissions and the number of vaccinations registered in three consecutive years. To our knowledge, there is only one article reporting the prevalence of urolithiasis in the dog population in Europe, specifically in Sweden and Norway (Wallerström and Wågberg, 1992). The calculation was made on the basis of data collected in a relatively long time period (26 years). The authors reported 23 to 24 cases/10,000 dogs within that period. This represented an average incidence of 0.88 to $0.92 / 10,000 /$ year. Our calculation revealed an approximately twice higher incidence, indicating that at least one dog potentially affected by urolithiasis should be expected among 5,600 individuals per year.

\section{Changes in the proportion of urolith types from 2001 to 2012}

Although a decreasing proportion of struvite uroliths was reported in longterm studies (Ling et al., 2003) and in global surveys (except for Australia and Oceania) (Lulich et al., 2013), the current study revealed an increasing frequency of struvite submissions. The percentage of calcium oxalate remained unchanged while the ratio of purine stones decreased. The prevalence of cystine stones showed inconsistent alteration probably due to the relatively small number of submissions (Table 4). The cause of the increase of struvite to calcium oxalate ratio in Hungary unlike observations from other parts of the world is not clear. The shift of that ratio can result from several factors (Wisener et al., 2010b). Previous studies did not really discuss whether the prevalence or the relative number of submissions had changed. As the physical appearance of struvite stones is characteristic in most cases, some practitioners might omit to submit such samples for analysis. This failure to submit physically well-recognisable struvite uroliths for analysis may result in apparently decreasing prevalence. Furthermore, the prevalence of struvite stones is probably associated with certain social and economic circumstances which are probably more expressed in the Eastern Euro- 
pean region, i.e. that early signs of urinary tract infections remain untreated or are mismanaged in the absence of expensive laboratory tests and medications.

\section{Evaluation of the effect of change in breed prevalence in the population especially in the breeds most affected by urolithiasis}

The proportion of the most affected breeds in the whole population (Yorkshire Terrier, West Highland White Terrier, Havanese, Bichon Frise, Beagle, Chihuahua, English Bulldog and Pug) increased about five times throughout the study period. Yorkshire Terriers and English Bulldogs were characterised by calcium oxalate in combination with purine and purine with cystine urolithiasis, respectively. The increasing ratio of these breeds adumbrates the increasing prevalence of calcium oxalate, purine and cystine urolithiasis. In addition, an expressed diminution of the proportion of Dalmatians, German Shepherds and Dachshunds, which were characterised by purine, struvite and cystine urolithiasis, respectively, was detected in the population. The change of breed proportions in the urolith database showed more or less the same tendency. As regards the breeds at risk, the prevalence of Yorkshire Terriers increased, while the proportion of Dalmatians and Black Russian Terriers markedly decreased during the study period. Whilst no sample was registered from the Havanese breed in the first years of the survey, 22 samples were submitted in the last two years, leading to an increase in the proportion from 0 to $4.4 \%$. Individuals of small breeds are exposed to environmental factors that can promote calcium oxalate stone formation (Lekcharoensuk et al., 2000). An increasing prevalence of Pug, Havanese, Chihuahua and Bichon Frise was also observed in the population. This trend predicts a plausible increase in calcium oxalate urolithiasis and a decrease in struvite, purine and cystine urolithiasis. While the proportion of Pekingese, Miniature Schnauzer and Puli decreased in the dog population, their prevalence consistently increased in the urolith database. The Pekingese and Miniature Schnauzer breeds have been associated with factors promoting the development of urolithiasis (Lekcharoensuk et al., 2000). The increasing expression of these factors is presumed in the background, but it remains unknown whether this was due to a certain genetic shift or environmental impacts. Concerning the Puli breed this change cannot be explained. The opposite change was observed for Basset Hounds and Chihuahuas. Basset Hounds are strongly susceptible to genetically determined cystinuria. A genetic shift in the gene pool, resulting in a decreasing frequency of the mutated gene(s) in this breed population of Hungary, may be responsible for that change. However, for Chihuahuas the observed change cannot be explained by these facts. 


\section{Limitations of the study}

Sample submission and the completeness of data. Although it is unlikely that all uroliths harvested from dogs were submitted to analysis, we presumed that almost all stones which were at all submitted for analysis in Hungary during the study period had been sent to the BUC. The estimation of the annual number of dogs in Hungary in the years 2010, 2011 and 2012 was based on the registered number of anti-rabies vaccinations for dogs, provided to the authors by the NFCSO. Although there is no evidence that all the dogs in Hungary were vaccinated and registered and all the uroliths that were formed and/or diagnosed were actually submitted, our findings could represent a correct estimation of the incidence of the disease as based on data collected from a reasonably large, welldelimited population possibly entirely covered by urolith analyses conducted by a single laboratory service.

Method. The method used for determining the chemical composition of urinary stones at the BUC was not able to differentiate calcium oxalate monohydrate from dihydrate in some cases. In order to obtain results comparable to those of other studies, the two chemical forms were considered together in the study. Xanthine-, dihydroxyadenine- or silica-containing uroliths were not detected in this study. As a similar finding was reported by other authors in in the Czech Republic, i.e. in an area geographically close to Hungary (Sosnar et al., 2005), the lack of these types of uroliths can be explained by their innumerably low frequency or by the deficiency of the method used for analysis. There were four cases in which composition of the urolith remained undetermined by our analytical technique.

Identification of the breed. The breed of an individual was identified by the referring veterinarian based on the dog's certification, physical appearance and/or the owner's statement. This might have resulted in a certain deviation, especially as regards the proportion of very common breeds (e.g. a German Shepherd-like crossbreed could be identified as a German Shepherd).

Population database. Two databases were processed to estimate the breed proportions at the start and at the end of the study period in the dog population from which the uroliths originated. The survey of the population was based on a non-selective dataset (data of all dogs living in Budapest), while the composition of the dog population was determined on the basis of a selective dataset (the records of microchipped dogs) at the end of the study period. Since microchip implantation was not fully compulsory in Hungary during the entire time of the study, some dogs may not have been included in the central register, which may have resulted in a possible alteration in the proportion of certain breeds. 


\section{Statistical analysis}

To estimate the risk of urolith formation in a certain breed or to evaluate the breed as a risk factor in the prevalence of a specific type of urolithiasis, the prevalence has to be compared to a reference group which is not affected by that particular risk factor in the same population. In this respect, crossbreed dogs affected by urolithiasis were selected as a reference group in this study, and their data were used for the calculation. Normally, a reference group must not be affected by any risk factor or must be affected equally by all risk factors. This could be true if all the known and unknown genetic or epigenetic factors had a homogeneous distribution in, or a balanced influence on, the crossbreed dogs. As this condition could not be proved, it could have an unpredictable impact on the OR results obtained in this study.

\section{Acknowledgements}

The authors thank the Hungarian Veterinary Chamber and the NFCSO for providing access to population data for the purpose of this study.

\section{References}

Bannasch, D. and Henthorn, P. S. (2009): Changing paradigms in diagnosis of inherited defects associated with urolithiasis. Vet. Clin. North Am. Small Anim. Prac. 39, 111-125.

Bende, B. and Németh, T. (2004): High prevalence of urate urolithiasis in the Russian Black Terrier. Vet. Rec. 155, 239-240.

Bende, B., Szabó, O. and Reiczigel, J. (2003): The dog population of Budapest at the millennium [in Hungarian, with English abstract]. Magyar Állatorvosok Lapja 125, 340-345.

Berényi, M. and Frang, D. (1989): News in ultramicrochemical stone analysis. In: Proceedings of the 1st European Symposium on Urolithiasis, Bonn. pp. 93-94.

Blavier, A., Sulter, A., Bogey, A., Novelli, K. and Billiemaz, B. (2012): Results of infrared spectrophotometry analysis of 1131 canine urinary stones, collected in France from 2007 to 2010 [in French]. Prat. Méd. Chir. Anim. Comp. 47, 7-16.

Brons, A.-K., Henthorn, P. S., Raj, K., Fitzgerald, C. A., Liu, J., Sewell, A. C. and Giger, U. (2013): SLC3A1 and SLC7A9 mutations in autosomal recessive or dominant canine cystinuria: A new classification system. J. Vet. Intern. Med. 27, 1400-1408.

Bruckner, A., Moreth, M. and Trendelenburg, C. (1989): Mikroskopisch-mikrochemische Harnsteinkomponentenanalyse: Vergleich mit Infrarotspektroskopie und Röntgendiffraktion. J. Clin. Chem. Clin. Biochem. 27, 730.

Del Angel-Caraza, J., Diez-Prieto, I., Pérez-García, C. C. C. and García-Rodríguez, M. B. B. (2010): Composition of lower urinary tract stones in canines in Mexico City. Urol. Res. 38, 201-204.

Henthorn, P. S., Liu, J., Gidalevich, T., Fang, J., Casal, M. L., Patterson, D. F. and Giger, U. (2000): Canine cystinuria: polymorphism in the canine SLC3A1 gene and identification of a nonsense mutation in cystinuric Newfoundland dogs. Hum. Gen. 107, 295-303.

Hesse, A. and Neiger, R. (2009): A Colour Handbook of Urinary Stones in Small Animal Medicine. Manson Publishing, Ltd., London. pp. 55-105. 
Houston, D. M. and Moore, A. E. (2009): Canine and feline urolithiasis: examination of over 50,000 urolith submissions to the Canadian Veterinary Urolith Centre from 1998 to 2008. Can. Vet. J. 50, 1263-1268.

Jones, B. R., Kirkman, J. H., Hogan, J. and Holmes, S. (1998): Analysis of uroliths from cats and dogs in New Zealand, 1993-96. New Zeal. Vet. J. 46, 233-236.

Jones, B. R., Omodo-Eluk, A. J., Larkin, H., Rogers, K. D. and Sperrin, M. (2001): Canine uroliths: analysis of uroliths from dogs in Ireland. Ir. Vet. J. 54, 629-632.

Karmi, N., Safra, N., Young, A. and Bannasch, D. L. (2010): Validation of a urine test and characterization of the putative genetic mutation for hyperuricosuria in Bulldogs and Black Russian Terriers. Am. J. Vet. Res. 71, 909-914.

Lekcharoensuk, C., Lulich, J. P., Osborne, C. A., Pusoonthornthum, R., Allen, T. A., Koehler, L. A., Urlich, L. K., Carpenter, K. A. and Swanson, L. L. (2000): Patient and environmental factors associated with calcium oxalate urolithiasis in dogs. J. Am. Vet. Med. Assoc. 217, 515-519.

Ling, G. V., Thurmond, M. C., Choi, Y. K., Franti, C. E., Ruby, A. L. and Johnson, D. L. (2003): Changes in proportion of canine urinary calculi composed of calcium oxalate or struvite in specimens analyzed from 1981 through 2001. J. Vet. Intern. Med. 17, 817-823.

Low, W. W., Uhl, J. M., Kass, P. H., Ruby, A. L. and Westropp, J. L. (2010): Evaluation of trends in urolith composition and characteristics of dogs with urolithiasis: 25,499 cases (19852006). J. Am. Vet. Med. Assoc. 236, 193-200.

Lulich, J. P., Osborne, C. A., Albasan, H., Koehler, L. A., Ulrich, L. M. and Lekcharoensuk, C. (2013): Recent shifts in the global proportions of canine uroliths. Vet. Rec. 172, 363-369.

Osborne, C. A., Lulich, J. P., Kruger, J. M., Ulrich, L. K. and Koehler, L. A. (2009): Analysis of 451,891 canine uroliths, feline uroliths, and feline urethral plugs from 1981 to 2007: perspectives from the Minnesota Urolith Center. Vet. Clin. North Am. Small Anim. Prac. 39, $183-197$.

Picavet, P., Detilleux, J., Verschuren, S., Sparkes, A., Lulich, J., Osborne, C., Istasse, L. and Diez, M. (2007): Analysis of 4495 canine and feline uroliths in the Benelux. A retrospective study: 1994-2004. J. Anim. Physiol. Nutr. 91, 247-251.

R Core Team (2013): A Language and Environment for statistical computing. R Foundation for Statistical Computing, Vienna, Austria, http://www.R-project.org

Roe, K., Pratt, A., Lulich, J., Osborne, C. and Syme, H. M. (2012): Analysis of 14,008 uroliths from dogs in the UK over a 10-year period. J. Small Anim. Pract. 53, 634-640.

Rogers, K. D., Jones, B., Roberts, L., Rich, M., Montalto, N. and Beckett, S. (2011): Composition of uroliths in small domestic animals in the United Kingdom. Vet. J. 188, 228-230.

Schenk, F., Rothenanger, E., Reusch, C. and Gerber, B. (2010): Analysis of 855 feline and 468 canine uroliths in Switzerland between 2002 and 2009. In: Proceedings of the 20th ECVIMCA Congress. p. 304.

Sosnar, M., Bulkova, T. and Ruzicka, M. (2005): Epidemiology of canine urolithiasis in the Czech Republic from 1997 to 2002. J. Small Anim. Pract. 46, 177-184.

Tobias, K. M. and Rohrbach, B. W. (2003): Association of breed with the diagnosis of congenital portosystemic shunts in dogs: 2,400 cases (1980-2002). J. Am. Vet. Med. Assoc. 223, 1636-1639.

Vrabelova, D., Silvestrini, P., Ciudad, J., Gimenez, J. C., Ballesteros, M., Puig, P. and Ruiz de Gopegui, R. (2011): Analysis of 2735 canine uroliths in Spain and Portugal. A retrospective study: 2004-2006. Res. Vet. Sci. 91, 208-211.

Wallerström, B. I. and Wågberg, T. I. (1992): Canine urolithiasis in Sweden and Norway: Retrospective survey of prevalence and epidemiology. J. Small Anim. Pract. 33, 534-539.

Wisener, L. V., Pearl, D. L., Houston, D. M., Reid-Smith, R. J. and Moore, A. E. (2010a): Risk factors for the incidence of calcium oxalate uroliths or magnesium ammonium phosphate uroliths for dogs in Ontario, Canada, from 1998 to 2006. Am. J. Vet. Res. 71, 1045-1054.

Wisener, L. V., Pearl, D. L., Houston, D. M., Reid-Smith, R. J. and Moore, A. E. (2010b): Spatial and temporal clustering of calcium oxalate and magnesium ammonium phosphate uroliths in dogs living in Ontario, Canada between 1998 and 2006. Prev. Vet. Med. 95, 144-151. 